LETTER TO THE EDITOR

\title{
Imatinib failure and response to dasatinib in a patient with chronic myeloid leukemia in blast crisis and a novel, nine-nucleotide BCR-ABL insertion mutation
}

Blood Cancer Journal (2013) 3, e104; doi:10.1038/bcj.2013.3; published online 8 March 2013

In chronic phase chronic myeloid leukemia (CML), the BCR-ABL kinase inhibitor imatinib leads to complete cytogenetic responses in the majority of cases. Resistance towards imatinib is associated with BCR-ABL kinase domain mutations, leading to structural changes that prevent imatinib from binding. ${ }^{1}$

In cases of failure towards imatinib treatment, second generation BCR-ABL kinase inhibitors such as dasatinib or nilotinib have demonstrated activity in CML. ${ }^{2}$ Both drugs are capable of suppressing imatinib-resistant, mutant forms of BCR-ABL. ${ }^{3}$ Most of the mutations in the $B C R-A B L$ gene mediating inhibitor resistance are point mutations, replacing single nucleotides. Splice mutations in BCR-ABL leading to deletion or insertion of nucleotide stretches have rarely been described. ${ }^{4}$

Here, we report on a patient with CML in blast crisis after imatinib failure and second-line treatment with dasatinib harboring a so far undescribed p.K294S_insFPQ mutation (g.68009_68010ins GTTCCCTC). A 37-year-old female patient initially presented with malaise, lymphadenopathy and splenomegaly. Her white blood cell count was $122.7 / \mathrm{nl}$ with $47 \%$ blasts. Bone marrow morphology showed $80 \%$ blast infiltration. Immunophenotyping revealed expression of CD34, HLA-DR, CD19, CD10, TdT and cyCD22. A Philadelphia chromosome-positive
CML with fusion transcript-type M-BCR (p210, b2a2) in primary lymphoid blast crisis was diagnosed with a $B C R-A B L / A B L$ ratio of $276 \%$.

Chemotherapy with daunorubicine and cytarabine and treatment with imatinib at a daily dose of $800 \mathrm{mg}$ was started. Imatinib was later reduced to 600 and $400 \mathrm{mg}$ due to pancytopenia. The $\mathrm{BCR}-\mathrm{ABL} / \mathrm{ABL}$ ratio only decreased to 139.6 and $48.2 \%$ on day 50 and 89 , respectively, with persistence of $10-15 \%$ bone marrow blasts and $17 \%$ blasts in the peripheral blood, indicating failure of treatment.

At day 99, BCR-ABL mutation analysis revealed a ninenucleotide insertion mutation (K294S_insFPQ) in the ABL kinase domain. Imatinib was discontinued, dasatinib was started at a dose of $100 \mathrm{mg}$ daily and three doses of vincristine and dexamethasone were given. On day $131, B C R-A B L / A B L$ ratio decreased to $13.8 \%$ and to $0.9 \%$ by day 173 under dasatinib treatment, indicating molecular response. Bone marrow analysis revealed $<5 \%$ blast cells. The level of hemoglobin was $8.8 \mathrm{~g} / \mathrm{dl}$, leukocytes $0.9 / \mathrm{nl}$ with $0 \%$ blasts and platelet $30 / \mathrm{nl}$, indicating complete remission with incomplete blood count recovery. BCR$A B L / A B L$ ratio had decreased to $13.8 \%$ under dasatinib treatment indicating molecular response. On day 173, the hemoglobin level was $8.6 \mathrm{~g} / \mathrm{dl}$, leukocytes $3.6 / \mathrm{nl}$ with $0 \%$ blasts and platelets $29 / \mathrm{nl}$, indicating complete hematologic remission with incomplete recovery of platelets. $B C R-A B L / A B L$ ratio was $0.9 \%$.

Allogeneic HLA-( $\left.A^{*}\right)$ antigen-mismatched peripheral blood stem cell transplantation (aSCT) was performed after conditioning with
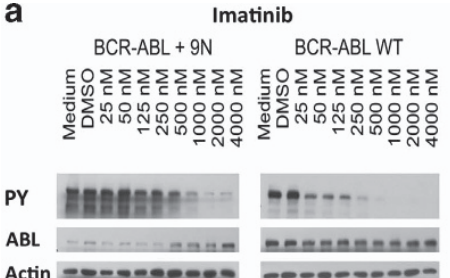

Actin $=0-000000$

$-0-0-0-0$

b

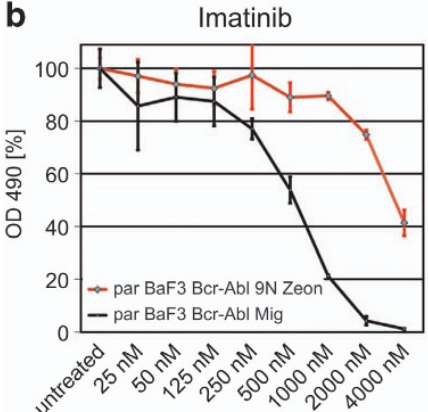

$$
\text { Dasatinib }
$$

BCR-ABL + 9N BCR-ABLWT
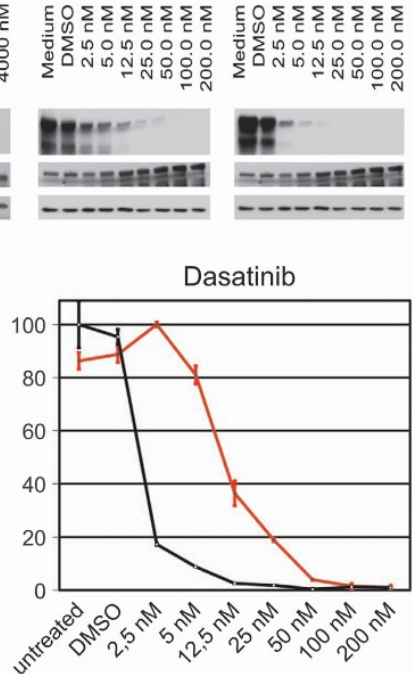
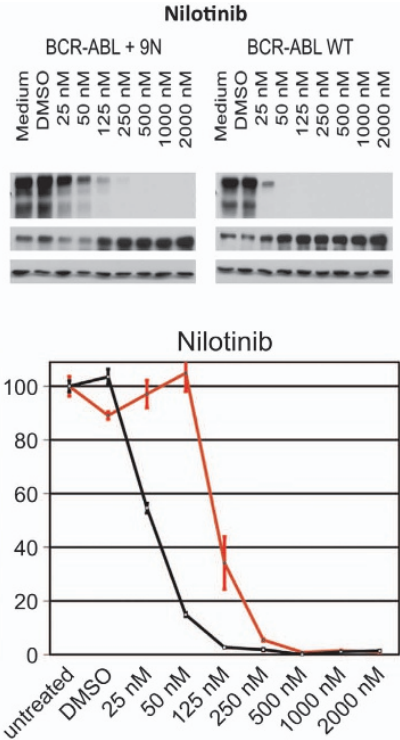

Figure 1. (a) Inhibition of autophosphorylation in BCR-ABL1 wild type and K294S_insFPQ demonstrated by western blot. Ba/F3 cells (BCR-ABL1 wild type and BCR-ABL1 K294S insFPQ) were cultivated in the presence of imatinib, dasatinib and nilotinib for $2.5 \mathrm{~h}$ at the indicated concentrations. (b) Assessment of cell growth. Proliferation was measured using an MTT-based method. Measures were taken as triplicates after $48 \mathrm{~h}$ of culture without and in the presence of inhibitors at the indicated concentrations. Values are expressed as representatives of growth inhibition from three independent experiments. One representative experiment is shown. Bars indicate \pm s.e. OD (optical density) \pm s.e.m. (standard error of mean). 
total body irradiation (at $12 \mathrm{~Gy}$ ), cyclophosphamide and antithymocyte globulin on day 205 after admission.

On day 100 after aSCT, complete molecular response with a donor chimerism of $100 \%$ was achieved. Hemoglobin level was $7.9 \mathrm{~g} / \mathrm{dl}$, leukocytes $1.7 / \mathrm{nl}$ and platelets were $24 / \mathrm{nl}$. One year after aSCT, graft versus host disease of the liver required the administration of steroids. CMV reactivation occurred, which was treated with ganciclovir. Fluorescence in situ hybridization analysis indicated complete cytogenetic response and BCR-ABL/ABL was $0 \%$ at different timepoints after aSCT (last result on day 757 after aSCT). Despite ongoing complete cytogenetic and molecular response, the bone marrow failed to completely recover. Transfusion-dependent thrombocytopenia and anemia persisted. On day 757 after aSCT, the bone marrow was still hypocellular with a donor chimerism of $97 \%$. On day 916 after aSCT, the patient died of septic multiorgan failure.

As shown in Figure 1a-b, the p.K294S_insFPQ mutation identified in this patient mediated a strong imatinib resistance and moderate resistance towards nilotinib and dasatinib. Cellular IC50 (halfmaximal inhibitory concentration) for imatinib was $4000 \mathrm{~nm}$, which corresponds to plasma trough levels at a dose of $800 \mathrm{mg}$ daily in humans. ${ }^{5}$ Accordingly, imatinib was not able to completely abrogate autophosphorylation in BCR-ABL/K294S_insFPQ. In contrast, dasatinib and nilotinib were able to completely inhibit cell growth and BCR-ABL autophosphorylation in K294S_insFPQexpressing cells at concentrations that correspond to plasma trough levels reported in treated patients (with dasatinib $70 \mathrm{mg}$ twice daily $100 \mathrm{~nm}$, nilotinib $400 \mathrm{mg}$ twice daily $1700 \mathrm{~nm})^{6}{ }^{6}$

Our patient displayed primary hematologic failure towards imatinib with persistence of peripheral blood blasts, which in this case can be attributed to the identified BCR-ABL/p.K294S_insFPQ mutation. Dasatinib and nilotinib both show residual activity against this particular mutation. In accordance, dasatinib was able to overcome imatinib resistance and able to induce major hematologic response and a decrease of $B C R-A B L / A B L$ ratio from 48.2 to $0.9 \%$ prior to aSCT. However, aSCT still bears the risk of nonrelapse mortality, even though the underlying disease is controlled.

Secondary mutations in BCR-ABL are mainly single-nucleotide point mutations of the kinase domain, ${ }^{1}$ deletion or insertion mutations are rare. A K294R point mutation has been identified in vitro to mediate weak imatinib resistance. ${ }^{7}$ A p.K294R insGG mutation recently was identified in a patient with blast crisis CML and imatinib resistance. ${ }^{8}$ Thus, more complex mutations such as insertion of several nucleotides adjacent to codon K294 that mediate strong resistance might require genetic instability as it is present in blast crisis. Mutations involving the ABL SH3-binding domain including K294 insertion mutations might retain sensitivity to dasatinib, as observed in our patient. This allows bridging to allogeneic stem cell transplantation, which to date still represents the only potential cure for advanced CML.

\section{CONFLICT OF INTEREST}

NvB and JD received honoraria from Novartis. SS declarespart ownership of the MLL Munich Leukemia Laboratory. All other authors declare no conflict of interest.

\section{ACKNOWLEDGEMENTS}

This work was supported by a grant to JD and NvB from the Bundesministerium für Bildung und Forschung (NGFNplus). We thank Peter Dreger, Universitätsklinikum Heidelberg, for his support.

M Sigl ${ }^{1,5}$, S Spoerl ${ }^{1,5}$, S Schnittger ${ }^{2}$, J Meissner ${ }^{3}$ C Rummelt ${ }^{1}, \mathrm{C} \mathrm{Peschel}^{1}$, J' Duyster ${ }^{4}, \mathrm{AD} \mathrm{Ho}^{3}$ and $\mathrm{N}$ von Bubnoff ${ }^{4}$

${ }^{1}$ III Medizinische Klinik und Poliklinik, Klinikum rechts der Isar, Technische Universität München, München, Germany;

${ }^{2}$ MLL, Münchner Leukämie Labor, München, Germany;

${ }^{3} \mathrm{~V}$ Medizinische Klinik und Poliklinik, Universitätsklinikum Heidelberg, Heidelberg, Germany and

${ }^{4}$ Klinik für Innere Medizin 1, Universitätsklinik Freiburg, Freiburg, Germany;

E-mail: nikolas.bubnoff@uniklinik-freiburg.de ${ }^{5}$ These authors contributed equally to this work.

\section{REFERENCES}

1 Apperley J. Mechanisms of resistance to imatinib in chronic myeloid leukaemia. Lancet Oncol 2007; 8: 1018-1029.

2 Hochhaus A, Baccarani M, Deininger M, Apperley J, Lipton J, Goldberg S et al. Dasatinib induces durable cytogenetic responses in patients with chronic myelogenous leukemia in chronic phase with resistance or intolerance to imatinib. Leukemia 2008; 22: 1200-1206.

3 Weisberg E, Manley P, Breitenstein W, Bruggen J, Cowan-Jacob S, Ray A. Characterization of AMN107, a selective inhibitor of native and mutant Bcr-Abl. Cancer Cell 2005; 7: 129-141.

4 De Melo V, Milojkovic D, Marin D, Apperley J, Nacheva E, Reid A. Deletions adjacent to $B C R$ and $A B L 1$ breakpoints occur in a substantial minority of chronic myeloid leukemia patients with masked Philadelphia rearrangements. Cancer Genet Cytogenet 2008; 182: 111-115.

5 Peng B, Hayes M, Resta D, Racine-Poon A, Druker B, Talpaz M et al. Pharmacokinetics and pharmacodynamics of imatinib in a phase I trial with chronic myeloid leukemia patients. J Clin Oncol 2004; 22: 935-942.

6 Kantarjian H, Giles F, Wunderle L, Bhalla K, O'Brien S, Wassmann B et al. Nilotinib in imatinib-resistant CML and Philadelphia chromosome-positive ALL. New Engl J Med 2006; 354: 2542-2551.

7 Azam M, Latek R, Daley G. Mechanisms of autoinhibition and STI-571/imatinib resistance revealed by mutagenesis of BCR-ABL. Cell 2003; 112: 831-843.

8 Sakai K, Ishikawa Y, Mori Y, Kobayashi M, Iriyama C, Ozawa Y et al. A novel insertion mutation of K294RGG within BCR-ABL kinase domain confers imatinib resistance: sequential analysis of the clonal evolution in a patient with chronic myeloid leukemia in blast crisis. Int J Hematol 2011; 93: 237-242.

(c) () $\Theta$ This work is licensed under a Creative Commons AttributionNonCommercial-NoDerivs 3.0 Unported License. To view a copy of this license, visit http://creativecommons.org/licenses/by-nc-nd/3.0/ 